\title{
On the Development of Rational Scaling Procedures for Liquid-Fuel Rocket Engines
}

\author{
S. S. PENNER ${ }^{2}$ \\ California Institute of Technology, Pasadena, Calif.
}

\begin{abstract}
A critical summary is presented of recent theoretical studies concerning similarity analysis and the scaling of liquid-fuel rocket engines. On the basis of this work, some suggestions are offered for an experimental program which has as its objective the development of rational scaling procedures.
\end{abstract}

\section{Introduction}

$\mathbf{T}$ $\mathrm{HE}$ construction of large liquid-fuel rocket engines has been hampered by the necessity of full-scale engine development programs. The experimental difficulties encountered with engines of different sizes are perhaps best illustrated by the occurrence of uncontrolled combustion oscillations, which are sometimes minimized or eliminated by artistic variations in injector design based on previous encounters with similar problems (1). ${ }^{3}$ In a survey paper concerned with experimental aspects of the rocket engine development program, Ross (2) has recently summarized the sort of concepts current among designers. Crocco (3) and Penner (4), commenting on this work, conclude that the science of engine scaling is still in an early phase of development.

During the last two years a concerted effort has been made to define the problem of engine scaling in scientific terms. Thus the important similarity parameters have been obtained (5) and shown to include the same dimensionless groups which are required for flow systems without chemical reactions, as well as two of the five similarity groups considered by Damköhler (6) in a classical paper on the scaling of chemical reactors. The dimensionless groups which must be maintained invariant in the scaling of chemical reactors (of which liquid-fuel rocket engines constitute a special class) are summarized in Section IIA.

Following Damköhler's procedure for chemical reactors, the problem of scaling for the steady internal aerothermochemistry of liquid-fuel rocket engines has been formulated (5). The method was then developed into an exact prescription for engine scaling on the assumption that the rocket combustion chamber may be considered to be a stirred reactor (7). The resulting scaling procedure may be shown to be consistent with a microscopic analysis of the combustion reactions, based on the assumption that the mass burning rate of the droplets is a linear function of the effective droplet diameter. Control over chemical conversion rate, which is essential for engine scaling with maintenance of exact similarity, is supposed to be obtained by control of droplet size, for example, through variation of the surface tension by

Presented at the ARS Fall Meeting, Buffalo, N. Y., Sept. 24$26,1956$.

1 The work described in this paper was supported by the U.S. Air Force, Office for Advanced Study, under Contract AF 18(603)-107 with the American Machine and Foundry Company and Jet Propulsion Research, Inc.

2 Associate Professor of Jet Propulsion, Daniel and Florence Guggenheim Jet Propulsion Center. Mem. ARS.

${ }^{3}$ Numbers in parentheses indicate References at end of paper. ${ }^{4}$ See Reference (7). the addition of suitable surface-active agents. Following Crocco's terminology (3), we shall refer to this scaling procedure as the P-T (Penner-Tsien) method. It is evident from the preceding remarks, relating to the physical model on which the P-T technique is based, that successful scaling of engines by this method can be accomplished only for bipropellant systems with greatly different volatilities (e.g., LOX - JP4 or $\mathrm{HNO}_{3}-\mathrm{NH}_{3}$ ).

The next important theoretical development is due to Crocco (3), who has succeeded in developing a rational scaling procedure for the steady operation and for high-frequency oscillations in the special case in which the chemical conversion time varies inversely with the first power of the pressure. This basic requirement may conceivably be met in practice for rocket motors in which the ignition time is long compared to the reaction time (i.e., particularly for nonhypergolic mixtures at relatively low pressures).

Engine development on the basis of the P-T model involves small-scale tests with motors utilizing very high injection velocities and fine sprays for the less volatile component. On the other hand, the procedure of Crocco requires model testing in rocket chambers operating at very high pressures.

We present in Section II a critical survey of theoretical studies related to the scaling of liquid-fuel rocket engines. In Section III we present some remarks on the development of an experimental program which is designed to furnish the needed basic data for the rational scaling of liquid-fuel rocket engines.

\section{Outline of Theoretical Studies}

We present in Section IIA a list of similarity parameters. Section IIB is devoted to a general formulation of engine scaling procedures. In Sections IIC and IID we consider, respectively, the development of the $\mathrm{P}-\mathrm{T}$ and Crocco rules. Included in Section IIC is a very simple argument due to Theodore von Kármán which is based on the idea that highfrequency oscillations can be avoided in large engines by maintaining the coupling between chemical and oscillation times at the same level as in a well-performing prototype, irrespective, for example, of the maintenance of similarity for the steady aerothermochemistry of motor operation.

\section{A Similarity Parameters for the Steady Aerothermo- chemistry of Motor Operation ${ }^{4}$}

Scaling criteria for homogeneous and heterogeneous chemical reactors may be obtained from the conservation equations for reacting gas mixtures. The complete set of similarity parameters has been shown to include the following dimensionless groups (5)

$$
\begin{aligned}
& \text { Reynolds number }=R e=\rho_{o} v_{o} L / \eta_{o} . \\
& \text { Schmidt number }=S c=\eta_{o} / \rho_{o} D_{o} \\
& \text { Prandtl number }=P r=c_{p},{ }_{o} \eta_{o} / \lambda_{o} \\
& \text { Mach number }=M=\sqrt{\rho_{o} v_{o}^{2} / \gamma_{o} p_{o}} .
\end{aligned}
$$


Froude number $=F r=v_{o}^{2} / g L \ldots \ldots \ldots \ldots[5]$

Damköhler's first similarity group $=\mathrm{D}_{\mathrm{I}}=\left(L / v_{0}\right) / t_{i}$.

Damköhler's third similarity group $=\mathrm{D}_{\mathrm{III}}=$

$$
q^{\prime} L / v_{o} c_{p, o} \vartheta_{o} t_{i} \ldots \ldots
$$

$\dot{\varphi}=\frac{1}{2} v_{o}^{2} /\left(c_{p}, o / \gamma_{o}\right) \vartheta_{o}$

and

$$
\gamma_{\circ}=c_{p, o} / c_{v}, \ldots \ldots \ldots \ldots \ldots \ldots[9]
$$

Here $\rho=$ density, $v=$ linear flow velocity, $L=$ a composite designation for all important lengths, $\eta=$ viscosity coefficient, $D=$ diffusion coefficient, $c_{p}=$ specific heat at constant pressure, $\lambda=$ thermal conductivity, $\gamma=$ ratio of the specific heat at constant pressure $\left(c_{p}\right)$ to the specific heat at constant volume $\left(c_{v}\right), g=$ constant gravitational acceleration, $t_{i}=$ characteristic conversion time of an important chemical species (e.g., of reaction product), ${ }^{5} q^{\prime}=$ standard specific enthalpy difference between reaction products and reactants, $\vartheta=$ temperature of the gas mixture; the subscript $o$ identifies suitably selected upstream reference conditions.

The characteristic conversion or reaction time $t_{i}$ is defined by the relation

$$
t_{i}=\rho Y_{i} / w_{i} \ldots \ldots \ldots \ldots \ldots \ldots
$$

where $w_{i}$ represents the net rate of mass change per unit volume produced by chemical reaction of the important chemical species $i$, and $Y_{i}$ denotes the corresponding weight or mass fraction. The parameter $D_{1}$ measures the ratio of the rate of change of $i$, produced by chemical reaction, to the rate of change of $i$ produced by convection. Similarly, $D_{I I}$ expresses the ratio of the rate of heat addition resulting from chemical reaction to the rate of heat addition associated with enthalpy flux by convection.

For low velocity flow problems involving fixed chemical reactants in the absence of external forces, results equivalent to Damköhler's (6) five similarity criteria are obtained (viz., only $[1,2,3,6$, and 7$]$ must remain invariant in order to maintain thermal, dynamic, and reaction kinetic similarity). Maintenance of complete chemical and reaction kinetic similarity in the gas stream automatically satisfies the boundary conditions for heat transfer to the chamber walls (i.e., the Nusselt heat transfer number is constant for fixed values of the Reynolds and Prandtl numbers) except in the case where surface-catalyzed chemical reactions play an important role. For surface-catalyzed reactions the boundary conditions remain similar if two new groups, analogous to those described by Equations [6, 7l, are introduced for the important chemical species reacting at the wall.

Our present state of ignorance concerning engine scaling is such that we do not know whether or not maintenance of constant Mach number is important. For this reason we shall follow Crocco (3) in treating scaling separately without consideration of $M$ (P-T rule and Crocco's second rule) and scaling for fixed $M$ without consideration of $\mathrm{D}_{1}$ or $\mathrm{D}_{\mathrm{II}}$ (Crocco's first rule). All of our discussion will be restricted to a fixed bipropellant mixture which is to be injected at the same temperature in both model (identified by the subscript $M o$ ) and large-scale (identified by the subscript $H$ for "Hauptausführung") experiments. The P-T and Crocco rules were developed without explicit reference to bipropellant systems. Since it may be instructive to focus attention on

5 The use of a single characteristic reaction time involves the implicit assumption that it is sufficient to restrict the discussion to consideration of the single chemical species $i$. This assumption appears to be justified for the following reason: If the physical states of two systems are similar, and if the time history of the important chemical species $i$ (e.g., of reaction product) is similar, then the entire complicated reaction scheme in the two systems will be similar. the physical variables involved, we utilize in Sections IIB and IIC a modified approach in which the special features of bipropellant operation are emphasized.

\section{B Formulation of Scaling Procedure for a Bipropellant Rocket Engine (Variable Mach Number)}

Consider a cylindrical bipropellant liquid-fuel rocket engine of length $l$ and diameter $d$. The chamber has a plane injection plate with regularly spaced injection orifices. The distance between fuel orifices is $d_{F}$, between oxidizer orifices $d_{o}$, and between fuel and oxidizer orifices $d_{F o}$. The fuel orifice diameter is $h_{F}$ and the oxidizer orifice diameter is $h_{O}$. Let $N_{F}$ and $N_{o}$ denote, respectively, the number of fuel and oxidizer injection orifices. Also $v_{F}$ and $v_{o}$ represent the corresponding fuel and oxidizer injection velocities.

The total mass flow rate in the large motor $H$ will be $n$ times the flow rate in the model $M o$ if

$$
\frac{\left(v_{o} h_{O}^{2} N_{O}\right)_{H}}{\left(v_{O} h_{O}^{2} N_{O}\right)_{M o}}=\frac{\left(v_{F} h_{F}^{2} N_{F}\right)_{H}}{\left(v_{F} h_{F}^{2} N_{F}\right)_{M o}}=n \ldots \ldots \ldots
$$

The quantity $n$ is thus the thrust ratio of the large motor $H$ with respect to the model $M o$.

For a given propellant system with fixed physicochemical parameters and for either fixed or variable chamber pressure, constancy of $P r$ and $S c$ is assured. Furthermore, $q^{\prime}$ is fixed and, for the same injection temperature, $\varphi_{0}$ is also fixed; therefore, constancy of $\mathrm{D}_{11 \mathrm{II}}$ is implied by constancy of $\mathrm{D}_{\mathrm{I}}$. The parameter $\gamma$ remains invariant and $\varphi$ is unimportant for small values of $v_{o}$. The Froude and Mach numbers will be ignored on the assumption that we are dealing with a low velocity flow problem in which acceleration effects are unimportant. The scaling requirements for fixed Mach number will be considered in Section IID. It is apparent that maintenance of exact similarity for the steady aerothermochemistry, subject to the previously noted simplifying assumptions, now requires only enforcement of the conditions of geometric similarity, of fixed $R e$ and of fixed $\mathrm{D}_{\mathrm{I}}$.

The requirements for geometric similarity of the chamber are

$$
\begin{aligned}
\frac{L_{H}}{L_{M o}}=\frac{\left(d_{F}\right)_{H}}{\left(d_{F}\right)_{M o}}=\frac{\left(d_{o}\right)_{H}}{\left(d_{o}\right)_{M o}}= & \frac{d_{H}}{d_{M o}}= \\
& \frac{\left(d_{F o}\right)_{H}}{\left(d_{F o}\right)_{M o}}=\frac{l_{H}}{l_{M o}}=n^{\prime} \ldots . .
\end{aligned}
$$

if the injector orifice diameters are small compared with all of the other important dimensions. Furthermore, if all of the distances are scaled in proportion, then the number of injection orifices is fixed, i.e.

$$
\frac{\left(N_{o}\right)_{H}}{\left(N_{O}\right)_{M o}}=\frac{\left(N_{F}\right)_{H}}{\left(N_{F}\right)_{M o}}=1
$$

Therefore, the over-all thrust relation given in Equation [11] becomes simply

$$
\frac{\left(v_{o} h_{O}^{2}\right)_{H}}{\left(v_{o} h_{O}^{2}\right)_{M_{o}}}=\frac{\left(v_{F} h_{F}^{2}\right)_{H}}{\left(v_{F} h_{F}^{2}\right)_{M_{o}}}=n \ldots \ldots \ldots \ldots
$$

Constant $R e$ will be maintained with respect to all chamber dimensions if

$$
\left(\rho_{o} v_{o} L\right)_{H}=\left(\rho_{o} v_{o} L\right)_{M o} .
$$

since $\eta_{0}$ is fixed. Here $v_{o}$ represents either $v_{F}$ or $v_{O}$ and $L$ is a composite designation for all chamber lengths. Finally, chemical similarity is maintained if

$$
\left(L / v_{o} t_{i}\right)_{H}=\left(L / v_{0} t_{i}\right)_{M_{o}}
$$

\section{Development of the P-T Rule (Fixed Chamber Pressure)}

For fixed chamber pressure, $\rho_{0}$ is constant, since the injection temperature $\vartheta_{o}$ has already been specified. There- 
fore, Equations $[12,13,14,15]$ may be combined to yield the relations

$$
\begin{gathered}
\frac{\left(v_{o}\right)_{H}}{\left(v_{o}\right)_{M o}}=\frac{\left(v_{F}\right)_{H}}{\left(v_{F}\right)_{M o}}=\frac{\left(v_{o}\right)_{H}}{\left(v_{o}\right)_{M o}}=\frac{1}{n^{\prime}} \ldots \ldots \ldots \\
\frac{\left(h_{F}^{2}\right)_{H}}{\left(h_{F}^{2}\right)_{M o}}=\frac{\left(h_{o}^{2}\right)_{H}}{\left(h_{o}^{2}\right)_{M o}}=n^{\prime} n \ldots \ldots \ldots \ldots \\
\frac{\left(t_{i}\right)_{H}}{\left(t_{i}\right)_{M o}}=\left(n^{\prime}\right)^{2} \ldots \ldots \ldots \ldots \ldots
\end{gathered}
$$

It has been emphasized previously (7) that a more detailed . prescription for rational motor scaling requires experimental determination of $t_{i}$ as a function of injector design, and of the physicochemical parameters affecting the problem, before Equations [16-18] can be used for the determination of the relation between $n$ and $n^{\prime}$. In the absence of quantitative information concerning the functional form of $t_{i}$, it is nevertheless of interest to illustrate the method of analysis by a simple example based on an oversimplified picture of the combustion processes.

\section{Maintenance of Similarity for Steady Operation With Uniform Combustion Throughout the Chamber Volume}

Observations of the interior of some rocket combustion chambers during operation show that the hot gases are under strong agitation and turbulence with eddy sizes of the order of the chamber dimensions. Hence it is reasonable to approximate these combustion chambers as homogeneous reactors. The mass rate of conversion $w_{i}$ of propellant for steady burning per unit volume is then equal to mass of propellant injected per unit time, divided by the volume of the combustion chamber, i.e.

$$
w_{i} \propto \frac{\rho_{O} v_{o} h_{O}^{2} N_{o}}{l d^{2}} \propto \frac{\rho_{F} v_{F} h_{F}^{2} N_{F}}{l d^{2}} \propto \frac{\rho_{o} v_{o} h^{2} N}{l d^{2}} .
$$

Since combustion occurs uniformly throughout the chamber volume, it follows that

$$
t_{i}=\frac{\rho_{0} Y_{i, o}}{w_{i}} \propto \frac{l d^{2} Y_{i, o}}{v_{0} h^{2} N}
$$

or, since $Y_{i, o}$ is fixed

$$
\frac{\left(t_{i}\right)_{H}}{\left(t_{i}\right)_{M o}}=\frac{\left(l d^{2}\right)_{H}}{\left(l d^{2}\right)_{M v}} \frac{\left(v_{\iota} h^{2} N\right)_{M o}}{\left(v_{o} h^{2} N\right)_{H}} \ldots
$$

Introduction of Equations [1], 12, and 18] into Equation [20] leads to the result

$$
\left(n^{\prime}\right)^{2}=\left(n^{\prime}\right)^{3}(1 / n)
$$

or

$$
n^{\prime}=n \text {. }
$$

In view of Equation [21] we now obtain the following basic results for maintenance of exact similarity with respect to the steady aerothermochemistry of internal motor operation (P-T rule)

$$
\begin{gathered}
(p)_{H}=(p)_{M o} \quad\left(\vartheta_{o}\right)_{H}=\left(\vartheta_{o}\right)_{M o} \\
\frac{\left(v_{o}\right)_{H}}{\left(v_{o}\right)_{M o}}=\frac{\left(v_{F}\right)_{H}}{\left(v_{F}\right)_{M o}}=\frac{\left(v_{o}\right)_{H}}{\left(v_{o}\right)_{M o}}=\frac{1}{n} \\
\frac{L_{H}}{L_{M o}} \equiv \frac{d_{H}}{d_{M o}} \equiv \frac{\left(d_{F}\right)_{H}}{\left(d_{F}\right)_{M o}}=\frac{\left(d_{o}\right)_{H}}{\left(d_{o}\right)_{M o}}=\frac{\left(d_{F o}\right)_{H}}{\left(d_{F o}\right)_{M o}}= \\
\frac{l_{H}}{l_{M o}}=\frac{\left(h_{F}\right)_{H}}{\left(h_{F}\right)_{M o}}=\frac{\left(h_{o}\right)_{H}}{\left(h_{o}\right)_{M o}}=n \\
\frac{\left(t_{i}\right)_{H}}{\left(t_{i}\right)_{M o}}=n^{2}
\end{gathered}
$$

According to the results summarized by the P-T rule, model tests should be made under the same pressure and temper- ature conditions as are required in the large engine. However, the injection velocity must be higher in the model than in the large engine by the factor $n$; all linear dimensions (including orifice spacing and orifice diameter) in the large engine must be increased in proportion to the desired thrust increase $n$; the time for conversion to reaction products must be increased by the factor $n^{2}$ in going from the model to the large engine.

It is apparent that the success of the entire scaling procedure rests on our ability to increase the characteristic reaction time proportionately with the square of the engine thrust. In this connection, we may ask whether the over-all considerations leading to the $\mathrm{P}-\mathrm{T}$ rule are consistent, for example, with the idea that the mass burning rate of a less volatile fuel (such as JP4 in a JP4-LOX mixture) is a linear function of the effective average droplet diameter $\bar{D}$. Here $\bar{D}$ is, in general, a function of the pressure drop across the injector orifices, of orifice diameter, and of the properties of the injected material. However, for heterogeneous diffusion flames in which the mass burning rate is a linear function of drop diameter, it is well known that the basic burning rate expression is

$$
\bar{D}_{t}^{2}=\bar{D}^{2}-K^{\prime} t
$$

where $\bar{D}_{t}$ is the drop diameter at time $t$ if the initial drop diameter is $\bar{D}$, and $K^{\prime}$ represents the evaporation constant. From Equation [22] it is apparent that the residence time $t_{\tau}$ in the chamber, corresponding to $\bar{D}_{t}=0$, is

$$
t_{r} \propto \bar{D}^{2}
$$

We now identify the conversion time $t_{i}$ with the residence time $t_{r}$ in a well-designed engine for combustion processes controlled by heterogeneous diffusion flames. It then follows from Equations $[18,21,23]$ that

$$
(\bar{D})_{H} /(\bar{D})_{M o}=n^{\prime}=n
$$

that is, the drop diameter for the assumed burning law specified by Equation [22] must be increased linearly with motor thrust. The desired control over droplet size may possibly be achieved by physicochemical methods (e.g., lowering of surface and interfacial tension) and is consistent with the idea of utilizing higher injection velocities in the model than in the large motor.

It is of interest to note that the P-T rule implies the relation $t_{i} \sim 1 / \Delta p$, where $\Delta p$ is the pressure drop across the injector orifice. This result follows directly from Equations $[16,23,24]$ if $v_{a}{ }^{2} \sim \Delta p$.

\section{Similarity of High-Frequency Oscillations}

We have no practical experience in evaluating the importance of maintaining similarity with respect to high-frequency oscillations either for engines which have or have not been properly scaled with respect to steady motor operation. There appear to be three possible points of view with regard to this problem. These are:

1 High-frequency oscillations will not develop if scaling has been done properly for steady motor operation.

2 Scaling should be done properly for steady operation but there will be sufficient intrinsic perturbations to produce instability. It is therefore important to scale with maintenance of a constant ratio of the chemical time $t_{i}$ to a wave propagation time $t_{w}$. Unstable motor operation is the result of coupling between the chemical and wave propagation times. We must maintain. synchronization between reaction and wave propagation processes below a fixed level. This result can presumably be achieved by fixing the ratio $t_{i} / t_{w}$ in $H$ at the same value as in $M o$ where no undesirable chamber oscillations occurred.

3 Scaling with maintenance of similarity for steady motor operation is unimportant. In order to prevent undesirable 
chamber oscillations it is only necessary to maintain the ratio $t_{i} / t_{w}$ invariant in going from a well-performing model to a large engine.

Implicit in the first point of view is the idea that similitude of the damping effects is sufficient to assure the absence of high-frequency oscillations. The damping effects, in turn, should be largely dependent on the value of $R e$ (which remains constant) and on the chemical evolution of the combustion processes (which is determined by $D_{1}$ ). Implicit in the third point of view is the notion that similitude of the driving forces for oscillations is the determining factor and that the damping processes are generally of similar intensity. Finally, the second point of view is based on the idea that high-frequency instability can be avoided only if the driving and damping forces for oscillations are maintained similar.

Depending on the point of view adopted, the P-T rule either is or is not useful since it is easily shown that the ratio $t_{i} / t_{w}$ does not remain constant. We proceed to demonstrate this fact on the basis of an analysis proposed independently by von Kármán and by Crocco (3).

For diffusion-controlled processes the conversion time has been shown to be proportional to the square of the scale factor for engine thrust. Thus

$$
\frac{\left(t_{i}\right)_{H}}{\left(t_{i}\right)_{M o}} \propto n^{2}
$$

On the other hand, $t_{w}$ is directly proportional to the geometric scale factor $n^{\prime}=n$ for constant sound velocity, i.e.

$$
\frac{\left(t_{w}\right)_{H}}{\left(t_{w}\right)_{M o}} \propto n
$$

Comparison of the preceding two relations shows immediately that $t_{i}$ and $t_{w}$ are not proportional for scaling according to the P-T rule. In other words, scaling according to the $\mathrm{P}-\mathrm{T}$ rule does not involve maintenance of similarity for possible high-frequency nonsteady motor operation.

It is a simple matter to obtain scaling criteria if the third of the points of view listed previously is adopted. For example, if heterogeneous gas-liquid diffusion flames are ratecontrolling, then the relation

$$
t_{i} / t_{w}=\text { const }
$$

leads to the requirement that

$$
t_{i}=t_{\tau} \propto n^{\prime}
$$

i.e., in view of Equation [23]

$$
\bar{D} \propto \sqrt{n^{\prime}} .
$$

In other words, the average droplet size for the low volatility component (e.g., JP4 in a JP4-LOX system) in a bipropellant engine should be scaled as the square root of the dimensions in order to maintain a fixed ratio $t_{i} / t_{w}$. As long as Equation [26] is satisfied it does not matter presumably how the other design variables are adjusted.

On the basis of the third point of view described above, Theodore von Karmán has suggested another scaling procedure, which may conceivably be applicable to bipropellant systems in which the fuel and oxidizer have similar volatilities (e.g., RFNA-AN). For processes of this type

$$
t_{i} \propto \frac{d_{F O^{2}}}{D^{*}}
$$

where $D^{*}$ is a suitably selected (binary) diffusion coefficient. On the other hand, for the high-frequency tangential mode of oscillation

$$
t_{w} \propto \frac{d}{a}
$$

where $a$ is the sound velocity. Hence, at constant pressure, the ratio $t_{i} / t_{w}$ will remain invariant if

$$
d_{F o} \propto \sqrt{d}
$$

i.e., if the distance between fuel and oxidizer orifices is scaled as the square root of the chamber diameter.

It is evident that one of the important problems which must be answered by an experimental program for engine scaling procedures is which of the points of view 1 to 3 is the correct one. Perhaps the first investigations should be designed to check the sufficiency or insufficiency of scaling merely with maintenance of constant ratios $t_{i} / t_{w}$. Although this method of approach requires an assumption about $t_{i}$, it is a relatively simple matter to test the scaling rules described in Equations [26, 28] for special motors in which the assumed rate-controlling reaction steps probably apply.

\section{Similarity of Cooling System, Low-Frequency Oscilla- tions, and Nozzle Design}

Crocco (3) has considered the design restrictions arising if similarity is maintained with respect to the cooling system, low-frequency oscillations, and nozzle design. His work is summarized in the next section in so far as it applies to the Crocco and P-T scaling procedures.

\section{Crocco's Analysis of Engine Scaling}

In the discussions presented in the preceding Sections IIB and IIC we have emphasized the relation between scaling parameters and motor design factors. We shall now simplify the discussion somewhat by dropping the distinction between design variables referring to the fuel and oxidizer individually. The analysis will again be restricted to a fixed propellant combination injected at a specified mixture ratio and at the same injection temperature.

Following Crocco's notation (3), we shall use the symbols

$$
\begin{gathered}
n_{d}=\frac{L_{H}}{L_{M o}}\left(\equiv n^{\prime}\right) \ldots \ldots \ldots \ldots \ldots . \\
n_{v}=\frac{v_{H}}{v_{M o}} \ldots \ldots \ldots \ldots \ldots \ldots \ldots
\end{gathered}
$$

and

$$
n_{t}=\frac{\left(t_{r}\right)_{H}}{\left(t_{r}\right)_{M o}}=\frac{n_{d}}{n_{v}} \ldots
$$

for the length, velocity, and residence time scale factors, respectively.

\section{Similarity Requirements for the Steady Aerothermo- chemistry}

Crocco has derived two rules, one,for fixed Mach number and one for an invariant Damköhler similarity group $D_{\mathrm{I}}$.

From the definition given for $M$ in Equation [4] it is apparent that, for a given propellant mixture and similar temperature history, $M$ will be constant if

$$
v_{H}=v_{M 0} \quad n_{v}=1 \quad \text { (Rule C.I) ......... }
$$

Furthermore, for fixed Reynolds number

$$
p_{H} L_{H}=p_{M o} L_{M o}
$$

whence it follows that

$$
p_{M o} / p_{H}=L_{H} / L_{M o}=n_{d}
$$

Equations [32, 33] identify the essential results of Crocco's Rule I for scaling with fixed $M$.

Next, Crocco assumes that the chemical conversion time varies inversely as the pressure raised to the $m$ th power, i.e.

$$
t_{i} \propto \frac{1}{p^{m}} \ldots \ldots \ldots \ldots \ldots \ldots
$$


Since combustion similarity can be maintained only if every residence time $t_{r}$ changes proportionally with the chemical time $t_{i}$, it follows that

$$
t_{i} \sim t_{r}
$$

Therefore, in view of Equations [31,34]

$$
n_{\iota}=\frac{\left(t_{r}\right)_{H}}{\left(t_{r}\right)_{M o}}=\left(\frac{p_{M o}}{p_{H}}\right)^{m}=\frac{n_{d}}{n_{v}} .
$$

and, for constant $R e$

$$
p_{H} v_{H} L_{H}=p_{M o} v_{M o} L_{M o}
$$

or

$$
\left(\frac{p_{M o}}{p_{H}}\right)^{m}=\left(\frac{v_{H}}{v_{M o}} \frac{L_{H}}{L_{M o}}\right)^{m}=\left(n_{v} n_{d}\right)^{m} \ldots \ldots .
$$

Comparison of Equations [35, 36] shows that

$$
\left(n_{v} n_{d}\right)^{m}=n_{d} / n_{r}=n_{t}
$$

or

$$
\begin{array}{r}
n_{v}=n_{d}^{(1-m) /(1+m)} \quad n_{t}=n_{d}^{2 m /(1+m)} \quad \text { (Rule C.II) } \ldots \\
\frac{p_{M o}}{p_{H}}=n_{d}^{2 /(1+m)} \quad(\text { Rule C.II }) \ldots \ldots \ldots
\end{array}
$$

Equations $[37,38]$ constitute the essential results of Crocco's Rule II. For $m=1$, Equations [32], [37], as well as Equations [33], [38], become identical; thus we maintain both $M$ and $\mathrm{D}_{\mathrm{I}}$ invariant at the same time. On the other hand, for $m=\infty$, Equations $[37,38]$ reduce to the P-T relation.

Whether or not the assumption $m=1$ (i.e., chemical conversion time inversely proportional to pressure) or $m=\infty$ (i.e., chemical conversion time independent of pressure) is a good approximation in any given case can only be determined experimentally. As has been emphasized in the discussion of the P-T rule, for diffusion-controlled processes the statement $m=1$ is certainly inadmissible. On the other hand, ignition delay times in rocket engines often vary roughly inversely with the chamber pressure. For rocket chambers in which the transient ignition processes constitute the major portion of the chemical conversion time it is therefore apparent that $m=1$ may constitute a fair approximation.

Crocco writes a composite expression for conservation of mass in the form

$$
\begin{array}{lc}
n=\frac{\left(p v d^{2}\right)_{H}}{\left(p v d^{2}\right)_{M o}} & \begin{array}{c}
\text { (i.e., } n \text { equals the ratio of flow } \\
\text { rates in the rocket chamber) }
\end{array} \\
n=\frac{\left(p r^{2}\right)_{H}}{\left(p r^{2}\right)_{M o}} & \begin{array}{c}
\text { (i.e., } n \text { equals the ratio of flow } \\
\text { rates through the nozzle throat) }
\end{array} \\
n=\frac{\left(v_{i} h^{2}\right)_{H}}{\left(v_{i} h^{2}\right)_{M o}} & \begin{array}{r}
\text { (i.e., } n \text { equals the ratio of flow } \\
\text { rates in the injection orifices) }
\end{array} \\
n=\frac{\left(v_{t} A_{t}\right)_{H}}{\left(v_{t} A_{t}\right)_{M o}} & \begin{array}{c}
\text { (i.e., } n \text { equals the ratio of flow } \\
\text { rates in the feed system) }
\end{array}
\end{array}
$$

Using the relations given previously, it is now easily shown that

$$
\begin{aligned}
& n=n_{d} \quad \text { (all rules).......... [40] } \\
& r_{H} / r_{M o}=n_{d} \quad \text { (Rule C.I) ....... [41] } \\
& r_{H} / r_{M o}=n_{d}^{(1 / 2)+1 /(1+m)} \quad(\text { Rule C.II) } \ldots \ldots[42] \\
& r_{H} / r_{M o}=n_{d}^{1 / 2} \quad(\mathrm{P}-\mathrm{T} \text { rule }) \ldots \ldots \ldots \ldots[43
\end{aligned}
$$

Furthermore, since $\left(v_{i}\right)_{H} /\left(v_{i}\right)_{M o}=n_{v}$

$$
\begin{aligned}
& h_{H} / h_{M o}=n_{d}^{1 / 2} \quad \text { (Rule C.I) ....... [44] } \\
& h_{H} / h_{M o}=n_{d}^{m /(1+m)} \quad \text { (Rule C.II). } \\
& h_{H} / h_{M o}=n_{d} \quad(\mathrm{P}-\mathrm{T} \text { rule }) \ldots \ldots \ldots \ldots
\end{aligned}
$$

Finally

$$
\left(v_{t}\right)_{H} /\left(v_{t}\right)_{M_{o}}=n_{t}\left(A_{t}\right)_{M_{o}} /\left(A_{t}\right)_{H} \text {. }
$$

Comparison of Equations [41] and [42] and of Equations [44] and [45] shows that Crocco's Rules I and II again become identical for $m=1$ and correspond to the case of no nozzle distortion, whereas the P-T rule (which is again obtained from C.II for $m=\infty$ ) always gives nozzle distortion. On the other hand, Equations [44-46] show that the orifice dimensions are scaled in proportion for the P-T rule but not for the Crocco rules. The practical significance of the indicated distortions cannot be assessed a.t the present time.

\section{Similarity of Cooling System}

Since the Reynolds and Prandtl numbers are fixed, the Nusselt heat transfer number also remains the same in the model and in the large engine. Hence the total heat which must be transferred to the motor walls, for chambers operating under similar conditions of temperature, pressure, and internal aerothermochemistry, must be directly proportional to the propellant consumption rate; this, in turn, varies linearly with the geometric scale factor, as has been shown in Equation [40]. Thus the total heat released and the total heat transfer to the motor walls must be directly proportional to $n=n_{d}$. Correspondingly the heat transfer per unit area varies as $n_{d} / n_{d}{ }^{2}=1 / n_{d}$.

Crocco has noted that complete similarity with respect to the cooling system will be maintained for constant coolant temperature and geometric wall scaling if the Nusselt number for the propellant(s) in the external flow also remains invariant, a condition which is easily achieved by maintaining geometric similarity for the coolant coils and constant Reynolds number in the coolant passages. The design of the cooling coils is closely related to the dynamic behavior of the feed system and must therefore be considered together with the problem of maintaining similarity for low-frequency stability (3).

\section{Similarity of Low-Frequency Stability}

Crocco (3) has discussed scaling procedures for a rigid and rigidly supported feeding system neglecting the compressibility of the propellants. Low-frequency stability depends on several parameters including the relative pressure drop $\Delta p / p(\Delta p=$ pressure drop across injection orifices; $p=$ steady operating pressure in the combustion chamber) and an inertia parameter which equals the ratio of the kinetic energy of the propellants in the feeding system to the work done on the propellants by the pressure drops during the residence time. The inertia parameter can always be adjusted by suitable selection of the lengths and cross-sectional areas of the feed lines (3).

Since the pressure drop across the injector orifices is proportional to the square of the flow velocities $v_{i}$, we find that

$$
\frac{\Delta p_{H}}{\Delta p_{M o}}=\frac{\left(v_{i}^{2}\right)_{H}}{\left(v_{i}^{2}\right)_{M \bullet}}=n_{v}^{2}
$$

whence

$$
\begin{aligned}
\epsilon \equiv \frac{(\Delta p / p)_{H}}{(\Delta p / p)_{M o}} & = \begin{cases}n_{d} & \text { for Rule C.I } \\
n_{d}{ }^{2(2-m) /(1+m)} & \text { for Rule C.II }\end{cases} \\
& =n_{d}{ }^{2} \quad \text { for the P-T rule..... }
\end{aligned}
$$

Thus the requirement $\epsilon=1$ cannot be met in practice except for Rule C.II in the unlikely case that $m=2$. For $m=1$ the two rules of Crocco again become identical but lead to relatively low reduced injector drops in the model. Crocco has suggested the use of injector orifices with appropriately poorer flow characteristics in the model than in the large engine in order to compensate for this effect (3).

In the P-T procedure the pressure drop is too large in the model, thereby necessitating the use of relatively poorer injection orifices in the large engine than in the model in order to maintain $\epsilon=1$. It is conceivable that this result can be obtained by physicochemical methods through appropriate 
changes in interfacial tension and may, in fact, be consistent with the modifications which must be introduced in order to achieve relatively high injection velocities and small drop diameters in the model tests.

For some further details concerning the design of the feed system and of its relation to low-frequency instability problems we refer to Crocco's original manuscript (3). In this connection it appears appropriate to emphasize the fact that it is generally much easier in practice to eliminate undesirable low-frequency oscillations than the high-frequency oscillations.

\section{High-Frequency Instability}

As has been noted in Section IIC-2, high-frequency instabilities can presumably be controlled, for otherwise similar operating conditions, if the ratio of the chemical time $t_{i}$ is fixed with respect to the wave propagation times $t_{w}$. But the latter are linear functions of the chamber dimensions for sound waves as well as for shock waves, provided the sound velocity remains invariant. Hence the similarity requirement for high-frequency instability may be written in the form

$$
\frac{\left(t_{i}\right)_{H}}{\left(t_{i}\right)_{M o}}=\frac{\left(t_{w o}\right)_{H}}{\left(t_{w}\right)_{M o}}=n_{d} \ldots \ldots \ldots \ldots \ldots
$$

Using Equation [34] and the results for the pressure ratios given in Equations [33 and 38], respectively, it is readily shown that

$$
\frac{\left(t_{i}\right)_{H}}{\left(t_{i}\right)_{M o}}=\left\{\begin{array}{ll}
n_{d^{m}} & \text { for Rule C.I } \\
n_{d^{2}}{ }^{2 m /(1+m)} & \text { for Rule C.II }
\end{array} \cdots \cdots\right.
$$

The result for the P-T rule has been obtained in Equation [18]. The P-T rule corresponds again to the limiting relation obtained for Crocco's Rule II if $m$ becomes very large.

Reference to Equations [49, 50] shows that the high-frequency stability of the system remains invariant as long as $m=1$, irrespective of whether constancy of the Mach number $M$ (Rule C.I) or of the first Damköhler similarity parameter $D_{I}$ (Rule C.II) is enforced. The deficiencies of the P.T rule, in so far as the high-\{requency stability requirement is concerned, have been noted previously in Section IIC-2.

Equation [49] expresses only the condition for similitude of the driving and coupling forces for oscillations. These forces producing oscillations must be balanced against the damping forces, of which the principal sources must be frictional losses. These, in turn, should be similar as long as the Reynolds number is constant. Damping associated with the oscillating gas flow through the nozzle is not similar unless the nozzles are scaled with maintenance of complete geometric similarity (which is always the case for Crocco's Rule I and applies also to Rule II if $m=1$ ). However, for a well-designed prototype it appears unlikely that damping through nozzle oscillations plays a dominant role.

\section{Some Critical Remarks Relating to Crocco's Scaling Rules}

It is apparent from the preceding discussion that Crocco has succeeded in deriving a consistent set of scaling rules only for the special case in which $m=1$. His proposed method requires model tests at elevated pressures, a procedure which raises formidable experimental problems. Furthermore, for a well-performing small rocket engine operating at elevated pressures, the effective $L^{*}$ required for acceptable chamber operation is probably practically independent of pressure. Therefore, the effective chemical time $t_{i}$ (which may be considered to be the sum of (a) an ignition time varying roughly inversely with the pressure and (b) a conversion time which is nearly independent of pressure) may well be nearly independent of pressure since, under steady operating conditions, the ignition time may be short compared to the con- version time. For these reasons we believe that it is unlikely that scaling with maintenance of similarity for all important operating characteristics is a practical possibility for real monopropellant or bipropellant liquid-fuel rocket engines. Nevertheless, Crocco's ingenious approach to the scaling problem deserves extensive experimental verification, because, at the very least, his work can be used as an intelligent guide in the interpretation of experimental findings.

\section{A Rational Experimental Program for the Study of Engine Scaling Procedures}

The survey given in Section II of theoretical studies relating to engine scaling procedures contains numerous obvious suggestions for an intelligent experimental program. We shall therefore content ourselves here with a few summary statements concerning some of the more promising ideas.

\section{A Experimental Studies on the Effect of Pressure on Operating Characteristics}

If $t_{i} \sim 1 / p$, Crocco's scheme is logically complete and suggests model tests on small engines operating at elevated pressures. The most economical procedure would appear to involve the design of models, using the scaling rules for $m=1$, based on known large engine performance data both for satisfactory and for unsatisfactory motors. ${ }^{6}$ We consider it more likely that this approach will prove successful for nonhypergolic bipropellant systems with components of roughly equal volatility (e.g., RFNA gasoline) than for either hypergolic mixtures or for propellants with greatly different volatilities.

\section{B Experimental Studies on the Effect of Injection Ve- locity and Droplet Size on Motor Performance}

The simplest way to answer the question concerning the importance of the steady aerothermochemistry would appear to be through the use of the P-T rule and the construction of small models, again on the basis of the known performance of large engines. In this connection, it is of primary importance to initiate studies on the control of spray distribution by physicochemical methods. As has been emphasized in the discussion given in Section IIC, the P-T rule is most likely to apply to systems in which the components have greatly different volatilities (e.g., JP4-LOX, RFNA- $\mathrm{NH}_{3}$ ).

\section{Diagnostic Modeling}

Since it appears likely that neither the Crocco rules nor the P-T rule offer a general answer to the scaling difficulties, the most fruitful method of approach is probably a more fundamental one than those suggested in Sections IIIA and IIIB. We must learn what are the really important similarity parameters and which dimensionless groups are of secondary importance. It is therefore necessary to perform tests in which (a) the driving force for high-frequency oscillations remains invariant both with and without constant Reynolds number, (b) only the damping (i.e., the steady aerothermochemistry and/or the Reynolds number) is unchanged, and (c) only the Mach number is constant, etc.

Correlated. with an experimental program on diagnostic modeling should be a concerted effort to define the phenomenological meaning of chemical times in rocket engines and their dependence on design variables, such as temperature, pressure, injector construction, etc. This work should ultimately lead to a new set of rules which, unlike those considered in the present discussion, have a firm experimental foundation and unquestionable practical value.

\footnotetext{
- According to a recent personal communication, Crocco is currently planning some experimental studies of this type.
} 


\section{Rational Scaling Procedures for Liquid-Fuel Rocket Engines}

(Continued from page 161)

\section{References}

1 Penner, S. S., and Datner, P. P., "Combustion in LiquidFuel Rocket Engines," Fifth Symposium (International) on Combustion, Reinhold, New York, 1955, pp. 11-29.

2 Ross, C. C., "Scaling of Liquid-Fuel Rocket Combustion Chambers," Paper presented at the Second AGARD Combus- tion Colloquium, Liége, Belgium, Dec. 1955, Butterworths Scientific Publications, London (in press).

3 Crocco, L. "Scaling of Liquid-Fuel Rocket Engines," ibid.

4 Penner, S. S., "Comments on Scaling of Liquid-Fuel Rocket Engines," ibid.

5 Penner, S. S., "Similarity Analysis for Chemical Reactors and the Scaling of Liquid-Fuel Rocket Engines," Combustion Researches and Reviews 1955, AGARD, Chap. 12, Butterworths Scientific Publications, London, 1955.

6 Damköhler, G., Z. Elektrochem., vol. 42, 1936, p. 846

7 Penner, S. S., "Models in Aerothermochemistry," Paper presented before International Symposium on Models in Engineering, Venice, Italy, Oct. 1955. 